\title{
多组变量的典型相关系数和典型相关变量 *
}

\author{
徐兴忠 \\ （青盘海洋大学应用数学系, 青离 266003)
}

\section{关链词 多组变量 协差阵 典型相关系数 典型相关变量}

\section{1 相关性度量}

设有 $K$ 组变量 $Y_{i}=\left(y_{i 1}, y_{i 2}, \cdots, y_{i p_{i}}\right)^{\prime}, i=1,2, \cdots, K, Y=\left(Y_{1}^{\prime}, Y_{2}^{\prime}, \cdots, Y_{K}^{\prime}\right)^{\prime}, Y$ 的协差 阵存在记为 $\Sigma$,

$$
\operatorname{cov} Y=\Sigma=\left(\begin{array}{lll}
\Sigma_{11} & \Sigma_{12} & \cdots \Sigma_{1 K} \\
\Sigma_{21} & \Sigma_{22} & \cdots \Sigma_{2 K} \\
\cdots & & \\
\Sigma_{K 1} & \Sigma_{K 2} & \cdots \Sigma_{K K}
\end{array}\right) .
$$

如何度量 $Y_{1}, Y_{2}, \cdots, Y_{K}$ 这 $K$ 组变量之间的相关性是本节研究的问题. 本文假定 $\Sigma_{i i}>0$, $i=1,2, \cdots, K$.

在 $K=2$ 时, 用

$$
\max _{\substack{a_{i}^{\prime} i_{i} a_{i}=1 \\ i=1,2 \\ i=1}} \operatorname{cov}\left(a_{1}^{\prime} Y_{1}, a_{2}^{\prime} Y_{2}\right)
$$

来度量 $Y_{1}$ 与 $Y_{2}$ 之间的相关性, 能得到重要的典型相关系数和典型相关变量的概念 ${ }^{[1]}$. 当 $K>2$ 时, $Y_{1}, Y_{2}, \cdots, Y_{K}$ 之间相关性的度量还未有好的定义, 如直接推广 $K=2$ 时的情形, 用

$$
\max _{\substack{a_{i} \sum_{i} a_{i}=1 \\ i=1,2, \cdots, K}} \sum_{1 \leqslant i<j \leqslant K} \operatorname{cov}\left(a_{i}^{\prime} Y_{i}, a_{j}^{\prime} Y_{j}\right)
$$

作为度量, 利用 Lagrange 乘数法, 这将导致多参数特征值问题而难以处理. 张尧庭 ${ }^{[2]}$ 给出了 度量

$$
1-\frac{|\Sigma|}{\prod_{i=1}^{K}\left|\Sigma_{i i}\right|} .
$$

张尧庭和朱晓冬 ${ }^{[3]}$ 从不同角度研究了这个度量的许多优良性质, 由于这个度量涉及到行列 式, 也不是很好处理. 上述两种方法最大的不足在于不能得到 $K=2$ 时, 建立典型相关分析所 必须的典型相关系数和典型相关变量这两个概念.

1995-07-17 收稿, 1995-11-08 收修改稿

*国家自然科学基金资助项目 
借鉴主成分分析的思想, 可以把第一主成分看成是一组变量的最大相关部分, 而它的方差 可作为这组变量的相关性度量. 因此, 若 $p_{1}=p_{2}=\cdots=p_{K}=1$, 则 $Y_{1}, Y_{2}, \cdots, Y_{K}$ 之间的相 关性可用下式来度量：

$$
\max _{t^{\prime} t=1} D\left(t^{\prime} Y\right) .
$$

对(1)式作适当的修正是必要的, 一是相关性对线性变换的不变性, 需对 $Y_{i}, i=1,2, \cdots, K$ 单 位化;二是(1)式的值不能保证在 $[0,1]$ 中, 因而也需要修正.

我们知道, 给定了 $K$ 个单位变量 $Y_{1}, Y_{2}, \cdots, Y_{K}$ 的协差阵 (相关阵) $R=\left(\rho_{i j}\right): K \times K$, 若 $t$ $\in R^{K}$ 为单位向量, 则

$$
D\left(\sum_{i=1}^{K} t_{i} Y_{i}\right)=\sum_{i=1}^{K} t_{i}^{2}+2 \sum_{1 \leqslant i<j \leqslant K} \rho_{i j} t_{i} t_{j} \leqslant\left(\sum_{i=1}^{K}\left|t_{i}\right|\right)^{2} \leqslant K,
$$

其中 $\left(t_{1}, t_{2}, \cdots, t_{K}\right)^{\prime}=t$. 由于 $\sum_{i=1}^{K} t_{i}^{2}=1$ 是方差部分, 不提供相关性, 所以, 应从(1)式中扣除. 设 $Y_{1}, Y_{2}, \cdots, Y_{K}$ 是 $K$ 个单位变量, 它们 的相关性度量可定义为

$$
\rho_{Y_{1} Y_{2} \cdots Y_{K}}=\frac{1}{K-1}\left(\max _{t=1} D\left(t^{\prime} Y\right)-1\right) \text {. }
$$

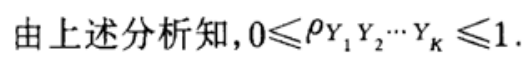

对于一般的 $p_{1}, p_{2}, \cdots, p_{K}$, 构造 $K$ 个单位变量 $a_{1}^{\prime} Y_{1}, a_{2}^{\prime} Y_{2}, \cdots, a_{K}^{\prime} Y_{K}$, 由(2)式来度量它 们之间的相关性, 并对 $a_{i}, i=1,2, \cdots, K$ 变化时求最大, 这个最大值就作为 $Y_{1}, Y_{2}, \cdots, Y_{K}$ 之 间相关性的度量. 因此, 有如下定义:

定义 1 称

$$
\rho_{Y_{1} Y_{2} \cdots Y_{K}}=\max _{\substack{a_{i} i_{i} a_{i}=1 \\ i=1,2, \cdots, K}} \sum_{i=1}^{K} \sum_{i=1}^{K} t_{i=1}^{2}=\frac{1}{K-1}\left(D\left(\sum_{i=1}^{K} t_{i} a_{i}^{\prime} Y_{i}\right)-1\right)
$$

为 $Y_{1}, Y_{2}, \cdots, Y_{K}$ 之间的相关系数.

性质 1 若 $K=2, p_{1}=p_{2}=1$, 则 $\rho_{Y_{1} Y_{2}}$ 等同于普通相关系数的绝对值.

证 记 $\Sigma=\left(\begin{array}{ll}\sigma_{11} & \sigma_{12} \\ \sigma_{21} & \sigma_{22}\end{array}\right)$, 由定义 1 ,

$$
\begin{aligned}
\rho_{Y_{1} Y_{2}}= & \max _{\substack{a_{i}^{2} a_{i i}=1 \\
i=1,2 \\
i=1,2}} \max _{t_{1}^{2}+t_{2}^{2}=1}\left(D\left(t_{1} a_{1} Y_{1}+t_{2} a_{2} Y_{2}\right)-1\right)= \\
& 2 \max _{\substack{t_{1}^{2}+t_{2}^{2}=1 \\
t_{1}}} t_{1} t_{2} \frac{\sigma_{12}}{\sqrt{\sigma_{11} \sigma_{22}}}=\frac{\left|\sigma_{12}\right|}{\sqrt{\sigma_{11} \sigma_{22}}} .
\end{aligned}
$$

性质 2 若 $K=2, p_{1}=1$, 则 $\rho_{Y_{1} Y_{2}}$ 等同于全相关系数的正平方根.

证 记 $\Sigma=\left(\begin{array}{cc}\sigma_{11} & \sigma^{\prime} \\ \sigma & \sigma_{22}\end{array}\right)$, 由定义 1 ,

$$
\begin{gathered}
\rho_{Y_{1} Y_{2}}=\max _{\substack{a_{1}^{2} \sigma_{11}=1 \\
a_{2}^{\prime} \Sigma_{22} a_{2}=1}} \max _{t_{1}^{2}+t_{2}^{2}=1}\left(D\left(t_{1} a_{1} Y_{1}+t_{2} a_{2}^{\prime} Y_{2}\right)-1\right)= \\
\frac{1}{\sqrt{\sigma_{11}}} \max _{a_{2}^{\prime} \Sigma_{22} a_{2}=1} \sigma^{\prime} a_{2}=\sqrt{\frac{\sigma^{\prime} \Sigma_{22}^{-1} \sigma}{\sigma_{11}}} .
\end{gathered}
$$




\section{2 典型相关系数和典型相关变量组}

现在求定义 1 给出的相关系数值, 这只需求 $D\left(\sum_{i=1}^{K} t_{i} a_{i}^{\prime} Y_{i}\right)$ 的极值. 令 $b_{i}=\Sigma_{j j}^{\frac{1}{2}} a_{i}, i=1,2$, $\cdots, K$, 记

$$
R=\left(R_{i j}\right), R_{i j}=\Sigma_{i i}^{-\frac{1}{2}} \Sigma_{i j} \Sigma_{j j}^{-\frac{1}{2}},
$$

则

$$
1+(K-1) \rho_{Y_{1} Y_{2} \cdots Y_{K}}=\max _{\substack{b_{i}^{\prime} b_{i}=1 \\ i=1,2, \cdots, K}} \max _{\sum_{i=1}^{K} t_{i}^{2}=1} \sum_{i, j=1}^{K} t_{i} t_{j} b_{i}^{\prime} R_{i j} b_{j}=\max _{c^{\prime} c=1} c^{\prime} R c .
$$

最后一个等式成立是由于

$$
\begin{gathered}
\left\{\left(t_{1} b_{1}^{\prime}, t_{2} b_{2}^{\prime}, \cdots, t_{K} b_{K}^{\prime}\right)^{\prime}: b_{i}^{\prime} b_{i}=1, i=1,2, \cdots, K, \sum_{i=1}^{K} t_{i}^{2}=1\right\}= \\
\left\{\left(c_{1}{ }^{\prime}, c_{2}{ }^{\prime}, \cdots, c_{K}{ }^{\prime}\right)^{\prime}=c: \quad c^{\prime} c=1\right\} .
\end{gathered}
$$

因此, 有如下定义:

定义 2 设 $R$ 由 (3) 式确定, $\lambda_{1} \geqslant \lambda_{2} \geqslant \cdots \geqslant \lambda_{r}$ 是 $R$ 的所有大于 1 的特征值, $c^{(1)}, c^{(2)}, \cdots$, $c^{(r)}$ 是对应的单位特征向量, 称 $\frac{\lambda_{i}-1}{K-1} \triangleq \rho_{i}$ 为 $Y_{1}, Y_{2}, \cdots, Y_{K}$ 的第 $i$ 个典型相关系数. 若记 $c^{(i)}=$ $\left(c_{1}^{(i)^{\prime}}, c_{2}^{(i)^{\prime}}, \cdots, c_{K}^{(i)^{\prime}}\right)^{\prime}$, 称 $t^{(i)}=\left(\sqrt{c_{1}^{(i)} c_{1}^{(i)}}, \sqrt{c_{2}^{(i)} c_{2}^{(i)}}, \cdots, \sqrt{c_{K}^{(i)} c_{K}^{(i)}}\right)^{\prime}$ 为第 $i$ 个相关权向量. 称

$$
\frac{c_{1}^{(i)^{\prime}} \Sigma_{11}^{-\frac{1}{2}} Y_{1}}{\sqrt{c_{1}^{(i)} c_{1}^{(i)}}}, \frac{c_{2}^{(i)^{\prime}} \Sigma_{22}^{-\frac{1}{2}} Y_{2}}{\sqrt{c_{2}^{(i)} c_{2}^{(i)}}}, \cdots, \frac{c_{K}^{(i)^{\prime}} \Sigma_{K K}^{-\frac{1}{2}} Y_{K}}{\sqrt{c_{K}^{(i)} c_{K}^{(i)}}}
$$

为 $Y_{1}, Y_{2}, \cdots, Y_{K}$ 的第 $i$ 组典型相关变量, $i=1,2, \cdots, K$, 若 $c_{j}^{(i)}=0$, 相应的变量舍去.

性质 3 (1) $\rho_{Y_{1} Y_{2} \cdots Y_{K}}=\rho_{1}$. (2) 典型相关变量组中每个变量都为单位变量. (3) 若记 $a_{j}^{(i)^{\prime}} Y_{j}, j=1,2, \cdots, K$ 为第 $i$ 组典型相关变量, $i=1,2, \cdots, K$, 则

$$
\begin{gathered}
t^{(i)^{\prime}}\left[\operatorname{cov}\left(\left(a_{1}^{(i)^{\prime}} Y_{1}, \cdots, a_{K}^{(i)^{\prime}} Y_{K}\right)^{\prime},\left(a_{1}^{(j)^{\prime}} Y_{1}, \cdots, a_{K}^{(j)^{\prime}} Y_{K}\right)^{\prime}\right)\right] t^{(j)}=\lambda_{i} \sigma_{i j}, \\
i, j=1,2, \cdots, K .
\end{gathered}
$$

证 由定义可得.

定理 1 若 $K=2$, 定义 2 中给出的典型相关系数和典型相关变量等同于经典的结果.

证 现 $R=\left(\begin{array}{cc}I & R_{12} \\ R_{21} & I\end{array}\right)$, 对 $R_{12}$ 作奇异值分解, $R_{12}=P \Lambda Q^{\prime}, P^{\prime} P=Q^{\prime} Q=I_{r}, \Lambda=$ $\operatorname{diag}\left(\rho_{1}, \rho_{2}, \cdots, \rho_{r}\right)>0$, 则经典的结果 ${ }^{[1]}$ 为: $\rho_{1}, \rho_{2}, \cdots, \rho_{r}$ 为典型相关系数, $\left(p_{i}{ }^{\prime} Y_{1}, q_{i}{ }^{\prime} Y_{2}\right)$ 为典 型相关变量, $i=1,2, \cdots, r$, 其中 $P=\left(p_{1}, p_{2}, \cdots, p_{r}\right), Q=\left(q_{1}, q_{2}, \cdots, q_{r}\right)$, 取 $\widetilde{P}, \widetilde{Q}$ 使 $(P, \widetilde{P})$ 和 $(Q, \widetilde{Q})$ 为正交阵, 则

$$
R=\left(\begin{array}{cc}
I & P \Lambda Q^{\prime} \\
Q \Lambda P^{\prime} & I
\end{array}\right)=\left(\begin{array}{cc}
P P^{\prime}+\widetilde{P} \widetilde{P}^{\prime} & P \Lambda Q^{\prime} \\
Q \Lambda P^{\prime} & Q Q^{\prime}+\widetilde{Q} \widetilde{Q}^{\prime}
\end{array}\right)=
$$




$$
\left(\begin{array}{cccc}
\frac{\sqrt{2}}{2} P & \widetilde{P} & 0 & -\frac{\sqrt{2}}{2} P \\
\frac{\sqrt{2}}{2} Q & 0 & \widetilde{Q} & \frac{\sqrt{2}}{2} Q
\end{array}\right)\left(\begin{array}{cccc}
I_{r}+\Lambda & 0 & 0 & 0 \\
0 & I & 0 & 0 \\
0 & 0 & I & 0 \\
0 & 0 & 0 & I_{r}-\Lambda
\end{array}\right)\left(\begin{array}{cc}
\frac{\sqrt{2}}{2} P^{\prime} & \frac{\sqrt{2}}{2} Q^{\prime} \\
\tilde{P}^{\prime} & 0 \\
0 & \widetilde{Q}^{\prime} \\
-\frac{\sqrt{2}}{2} P^{\prime} & \frac{\sqrt{2}}{2} Q^{\prime}
\end{array}\right) \triangleq T \Delta T^{\prime},
$$

这里 $\Delta$ 为对角阵, $T$ 为正交阵. 所以, 由定义 2 知, 相关权向量总是 $\left(\frac{\sqrt{2}}{2}, \frac{\sqrt{2}}{2}\right)$, 而典型相关系 数和典型相关变量与经典结果等同.

定理 2 对 $\Sigma$ 作秩分解 $\Sigma=F^{\prime} F$, 记 $F=\left(F_{1}, F_{2}, \cdots, F_{p_{K}}\right)$, 令 $Q_{i}=F_{i}\left(F_{i}^{\prime} F_{i}\right)^{-1} F_{i}^{\prime}, i=1$, $2, \cdots, K, Q=Q_{1}+Q_{2}+\cdots+Q_{K}$, 设 $\lambda_{1} \geqslant \lambda_{2} \geqslant \cdots \geqslant \lambda_{r}>1$ 是 $Q$ 的所有大于 1 的特征值, $d_{1}$, $d_{2}, \cdots, d_{r}$ 是对应的 $Q$ 的特征向量, 则 $\frac{\lambda_{i}-1}{K-1}$ 是第 $i$ 个典型相关系数, $\frac{d_{i}^{\prime} F_{j}\left(F_{j}^{\prime} F_{j}\right)^{-1} Y_{j}}{\sqrt{d_{i}^{\prime} Q_{j} d_{i}}}, j=1$, $2, \cdots, K$ 是第 $i$ 组典型相关变量, $i=1,2, \cdots, r$, 其中若 $d_{i}^{\prime} Q_{j} d_{i}=0$, 相应的典型变量舍去.

证 现 $R_{i j}=\left(F_{i}^{\prime} F_{j}\right)^{-\frac{1}{2}} F_{i}^{\prime} F_{j}\left(F_{j}^{\prime} F_{j}\right)^{-\frac{1}{2}}, i, j=1,2, \cdots, K$, 即

$$
R=\left(\begin{array}{c}
\left(F_{1}^{\prime} F_{1}\right)^{-\frac{1}{2}} F_{1}^{\prime} \\
\left(F_{2}^{\prime} F_{2}\right)^{-\frac{1}{2}} F_{2}^{\prime} \\
\vdots \\
\left(F_{K}^{\prime} F_{K}\right)^{-\frac{1}{2}} F_{K}^{\prime}
\end{array}\right)\left(F_{1}\left(F_{1}^{\prime} F_{1}\right)^{-\frac{1}{2}}, F_{2}\left(F_{2}^{\prime} F_{2}\right)^{-\frac{1}{2}}, \cdots, F_{K}\left(F_{K}^{\prime} F_{K}\right)^{-\frac{1}{2}}\right) .
$$

若 $c$ 是 $R$ 的对应于特征值大于 1 的特征向量, 则 $c \in \mathcal{M}(R)$, 即存在 $d$, 使

$$
c=\left(F_{1}\left(F_{1}^{\prime} F_{1}\right)^{-\frac{1}{2}}, F_{2}\left(F_{2}^{\prime} F_{2}\right)^{-\frac{1}{2}}, \cdots, F_{K}\left(F_{K}^{\prime} F_{K}\right)^{-\frac{1}{2}}\right)^{\prime} d .
$$

因此, $R c=\lambda c$ 等价于 $Q d=\lambda d$, 由定义 2 知定理结论成立.

定理 2 给出了一种求典型相关系数和典型相关变量组的新方法, 重要的是它有利于对典 型相关系数的研究.

定理 3 (1) $\rho_{Y_{1} Y_{2} \cdots Y_{K}}=0$ 的充要条件是 $Y_{1}, Y_{2}, \cdots, Y_{K}$ 不相关. (2) $\rho_{Y_{1} Y_{2} \cdots Y_{K}}=1$ 的充 要条件是存在 $a_{i}, a_{i}^{\prime} \Sigma_{i i} a_{i}=1, i=1,2, \cdots, K$ 使

$$
P\left(a_{1}^{\prime}\left(Y_{1}-E Y_{1}\right)=a_{2}^{\prime}\left(Y_{2}-E Y_{2}\right)=\cdots=a_{K}^{\prime}\left(Y_{K}-E Y_{K}\right)\right)=1 \text {. }
$$

证 (1) 若 $Y_{1}, Y_{2}, \cdots, Y_{K}$ 不相关, 则 $R=I$, 由定义知 $\rho_{Y_{1} Y_{2} \cdots Y_{K}}=\rho_{1}=0$. 反之, 若 $\rho_{1}=$ 0 , 则由定理 $2, Q$ 的最大特征根 $\lambda_{1}=1$. 因此, $Q \leqslant I$, 从而 $Q_{i} Q Q_{i} \leqslant Q_{i}^{2}=Q_{i}, i=1,2, \cdots, K$, 等 价于

$$
Q_{i}\left(\sum_{j \neq i} Q_{j}\right) Q_{i}=\sum_{j \neq i} Q_{i} Q_{j} Q_{i} \leqslant 0 .
$$

由 $Q_{i} Q_{j} Q_{i}$ 的非负定性知, $Q_{i} Q_{j}=0, i \neq j, i, j=1,2, \cdots, K$, 等价于 $\Sigma_{i j}=0, i \neq j, i, j=1,2$, $\cdots, K$.

(2) 若(4)式成立, 则 


$$
\begin{aligned}
1 \geqslant \rho_{Y_{1} Y_{2} \cdots Y_{K}} \geqslant & \frac{1}{K-1}\left(D\left(\sum_{i=1}^{k} \frac{1}{\sqrt{K}} a_{i}^{\prime} Y_{i}\right)-1\right)= \\
& \frac{1}{K-1}\left(\frac{1}{K} D\left(K a_{1}^{\prime} Y_{1}\right)-1\right)=1 .
\end{aligned}
$$

所以, $\rho_{Y_{1} Y_{2} \cdots Y_{K}}=1$. 反之, 若 $\rho_{Y_{1} Y_{2} \cdots Y_{K}}=\rho_{1}=1$, 由定理 2 知, $Q$ 有一个特征值为 $K$, 即存在 $d$ 使 $Q d=K d$, 不妨设 $d$ 为单位向量, 则

$$
d^{\prime} Q_{1} d+d^{\prime} Q_{2} d+\cdots+d^{\prime} Q_{K} d=d^{\prime} Q d=K d^{\prime} d=K \text {. }
$$

由于 $d^{\prime} Q_{i} d \leqslant d^{\prime} d=1, i=1,2, \cdots, K$, 上式成立必有 $d^{\prime} Q_{i} d=1$. 又由于 $Q_{i}{ }^{\prime}$ 是正交投影阵, 必 然地有 $d \in \mathscr{M}\left(Q_{i}\right)$, 即 $Q_{i}=d, i=1,2, \cdots, K$.

现考虑 $\left(d^{\prime} F_{1}\left(F_{1}{ }^{\prime} F_{1}\right)^{-1} Y_{1}, d^{\prime} F_{2}\left(F_{2}{ }^{\prime} F_{2}\right)^{-1} Y_{2}, \cdots, d^{\prime} F_{K}\left(F_{K}{ }^{\prime} F_{K}\right)^{-1} Y_{K}\right)$, 它的协方差阵 的所有元素都为 1 . 所以, 若令 $a_{i}=\left(F_{i}^{\prime} F_{i}\right)^{-1} F_{i}^{\prime} d$, 则 $a_{i}^{\prime} \Sigma_{i i} a_{i}=1, i=1,2, \cdots, K$, 且 (4) 式成 立. 定理证毕.

最后指出, 本文的研究是初步的, 还有许多工作需要去做, 如:

(1) $\rho_{Y_{1} Y_{2} \cdots Y_{K}}$ 是否具有文献 $[2,3]$ 中所述的性质?

(2) 一般地, 实际中的 $\Sigma$ 是未知的, 需用样本协方差阵 $\hat{\Sigma}$ 去估计, 相应的 $\hat{\rho}_{1}, \hat{\rho}_{2}, \cdots, \hat{\rho}_{r}$ 的 分布是什么?

(3) 在 $K=2$ 时, 相关权向量总是 $\left(\frac{\sqrt{2}}{2}, \frac{\sqrt{2}}{2}\right)^{\prime}$, 表明两组变量的地位是等同的, 在 $K>2$ 时, 相关权向量的分量表示各组变量在典型相关变量组中的贡献, 在用 $\hat{\Sigma}$ 替代 $\Sigma$ 后, 它们的分布 是什么?

（4）设 $\mathbb{R}^{n}$ 中有 $K$ 个子空间 $V_{1}, V_{2}, \cdots, V_{K}$, 它们的维数分别为 $p_{1}, p_{2}, \cdots, p_{K}$, 正交投影 阵分别为 $Q_{1}, Q_{2}, \cdots, Q_{K}$. 设 $p=\max \left\{p_{1}, p_{2}, \cdots, p_{K}\right\}, Q=Q_{1}+Q_{2}+\cdots+Q_{K}, Q$ 的最大特 征根为 $\lambda$, 第 $p$ 大特征根是 $\mu$, 则称 $\frac{\lambda-1}{K-1}$ 为这 $K$ 个子空间的贴近度, $1-\frac{\mu-1}{K-1}=\frac{K-\mu}{K-1}$ 为这 $K$ 个子空间的分离度. 这种定义是否合理?

\section{参考文献}

\footnotetext{
1 方开泰. 实用多元统计分析. 上海: 华东师范大学出版社, 1989

2 张尧庭. 多组随机变量相关性的度量. 曲皁师范学院学报, 1984, 3: 1 6

3 张尧庭, 朱晓冬. 随机向量相关性的度量. 应用概率统计, 1988, 4(1):27 34
} 